\section{Inserção e atuação do profissional de educação física na atenção básica à saúde: revisão sistemática}

\section{Integration and performance of Physical Education Professional on primary health care in Brazil: systematic review}

José Damião Rodrigues ${ }^{1,2,3}$

Daniela Karina da Silva Ferreira 2,3

Patrícia Andréia da Silva ${ }^{2,4}$

Iraquitan de Oliveira Caminha ${ }^{2,4}$

José Cazuza de Farias Junior ${ }^{2,5}$

\section{Resumo}

Este estudo teve como objetivo analisar estudos originais sobre inserção e atuação do Profissional de Educação Física (PEF) na atenção básica à saúde no Brasil. Trata-se de uma revisão sistemática que analisou os aspectos: i) processo de inserção do PEF na atenção básica à saúde; ii) atividades desenvolvidas pelos $\mathrm{PEF}$; iii) principais potencialidades e fragilidades na atuação do PEF na atenção básica à saúde. Efetuaram-se buscas nas bases de dados eletrônicas LILACS, MEDLINE, COCHRANE, Google acadêmico e em listas de referências dos artigos identificados. Foram incluídos artigos originais publicados entre janeiro de 2005 e outubro de 2011, em português e inglês. Nas buscas foram utilizados os descritores: "saúde pública", "educação física", "atenção básica à saúde", "programa saúde da família”, os termos "estratégia saúde da família”, "núcleo de apoio à saúde da família", "profissional de educação física”. Foram identificados 5.793 estudos, nove atenderam aos critérios de inclusão previamente estabelecidos. Identificou-se que a inserção do PEF na atenção básica à saúde ocorre principalmente por contrato temporário; as atividades mais desenvolvidas são caminhada, fortalecimento muscular e atividades lúdicas; destaca-se como potencialidade na atuação do PEF o fato de contribuir para melhoria dos níveis de saúde e qualidade de vida dos usuários e como fragilidade a formação profissional pouco direcionada para o trabalho interdisciplinar e com grandes grupos populacionais. Conclui-se que a atual formação do PEF não contempla adequadamente sua atuação na atenção básica à saúde. Entretanto, esse é o único profissional com formação para orientar a prática de atividades físicas.

\section{Palavras-chave}

Saúde pública; Educação física; Atenção básica à saúde; Estratégia saúde da família.

\begin{abstract}
This study aimed to analyze original studies on integration and performance of Physical Education Professional (PEP) on primary health care in Brazil. It is a systematic review that examined aspects: i) insertion of the PEP in primary health care, ii) activities undertaken by the PEP, iii) the main strengths and weaknesses in the performance of the PEP in primary care. We carried out searches in electronic databases LILACS, MEDLINE, COCHRANE, Google scholar and reference lists of identified articles. We included original articles published between January 2005 and October 2011, in Portuguese and English. In the searches were used descriptors: "public health", "physical education", "primary care", "Family Health Program", the words "family health strategy," "core support to family health," "physical education professional." We identified 5793 studies, nine met the inclusion criteria previously established. In the results, we found that the insertion of the PEP in primary care is due mainly to temporary contract, the activities are more developed walking, strengthening exercises and recreational activities; stands out as the potential role of PEP in the fact contribute to raising standards health and quality of life of users and how fragile a little directed training for interdisciplinary work and with large population groups. We conclude that the actual formation of the PEF fails adequately its role in primary health care. However, this is the only professional trained to guide the practice of physical activities.
\end{abstract}

\section{Keywords}

Public health; Physical education; Primary health care; Family health strategy.
Rev Bras Ativ Fis Saúde p. 5-15 DOI: http://dx.doi.org/10.12820/23171634.2013v18n1p5

1 Universidade Estadual da Paraiba, Campina Grande, PB, Brasil.

2 Programa Associado de Pós-Graduação em Educação Física UPE/UFPB, Universidade Federal da Paraíba, João Pessoa, PB, Brasil.

3 Laboratório de Estudos e Pesquisas em Atividade Física e Saúde, Universidade Federal da Paraíba, João Pessoa, PB, Brasil.

4 Laboratório de Estudos sobre Corpo, Estética e Sociedade - LAISTHESIS, Universidade Federal da Paraíba, João Pessoa, PB, Brasil.

5 Grupo de Estudos e Pesquisas em Epidemiologia da Atividade Física - GEPEAF Universidade Federal da Paraíba, João Pessoa, PB, Brasil. 


\section{INTRODUÇÃO}

A saúde pública no Brasil vem passando por várias mudanças desde 1970, dentre elas a reforma sanitária e a criação do Sistema Único de Saúde (SUS $)^{1}$ regulamentado pela lei 8.080/1990². Essa reforma buscou o reconhecimento da saúde como direito social e trouxe um novo modelo de assistência pública à saúde, caracterizada pela inserção do princípio da universalização das ações de saúde, descentralização, integralidade e regionalização no atendimento ${ }^{3-6}$.

Com o intuito de consolidar o SUS foi criado em 1994 o Programa Saúde da Família (PSF) através da Portaria Ministerial no 692/947, cujo foco principal é a família no seu contexto físico e social objetivando a promoção da saúde ${ }^{3-6,8}$. O PSF teve seu status modificado de programa para Estratégia Saúde da Família $(\mathrm{ESF})^{9}$ devido às limitações do termo programa. A ESF é composta por uma equipe multiprofissional formada por médico, enfermeiro, auxiliares de enfermagem e agentes comunitários de saúde ${ }^{10}, 11$, com esforços dirigidos no cuidar não só do usuário, mas também de sua família, através de planejamento direcionado à realidade da comunidade adstrita ${ }^{3}$.

Como a ESF não conseguiu suprir a demanda com a devida cobertura à atenção básica à saúde ${ }^{12}$, fez-se necessário qualificar a assistência e estabelecer melhor a demanda programada ${ }^{13}$. Para isso, o Ministério da Saúde criou os Núcleos de Apoio à Saúde da Família (NASF) através da Portaria G.M no 154/2008 ${ }^{14}$. Com a criação dos NASF em 2008, passou-se a ter maior ênfase e apoio ao cumprimento dos objetivos preconizados pelo SUS e ESF por meio de uma equipe multiprofissional, que inclui médico acupunturista, assistente social, profissional de educação física (PEF), farmacêutico, fisioterapeuta, fonoaudiólogo, médico ginecologista, médico homeopata, nutricionista, médico pediatra, psicólogo, médico psiquiatra e terapeuta ocupacional ${ }^{14}$. Em 2011 foram acrescentados, a essa equipe, médico geriatra, médico internista (clínica médica), médico do trabalho, médico veterinário, profissional com formação em arte e educação e profissional de saúde sanitarista ${ }^{15}$. Essa equipe visa oferecer suporte à promoção, manutenção e reabilitação da saúde, bem como o tratamento das doenças na comunidade adstrita ${ }^{16}$.

O PEF foi reconhecido como profissional de saúde através da Resolução CNS - $\mathrm{N}^{\circ} 218$, de 6 de março de $1997^{17}$, principalmente devido às mudanças no perfil de morbidade e mortalidade, caracterizadas pelo predomínio das Doenças Crônicas Não Transmissíveis (DCNT), evidenciando-se a inatividade física como um dos quatro principais fatores de risco para esse grupo de causas ${ }^{18}$. Paralelamente, evidências de estudos epidemiológicos têm demonstrado que a prática de atividade física está associada a menores riscos de morte por todas as causas e por DCNT, aumento da expectativa de idade e melhor qualidade de vida ${ }^{10,19}$. Nesse sentido, em 2006, foi aprovada a Política Nacional de Promoção da Saúde que inseriu a prática de atividade física na estratégia de promoção de saúde, reforçando a importância do estilo de vida fisicamente ativo ${ }^{12}$. Essa conjuntura culminou com a inserção do PEF na atenção básica à saúde, especialmente no NASF, na Academia da Cidade e em projetos que visem à promoção, proteção e reabilitação da saúde 2 . Desse modo, a participação do $\mathrm{PEF}$ na atenção básica à saúde é uma das estratégias para aumentar a prática de atividade física na população e minimizar os riscos ocasionados pela inatividade física ${ }^{2,11,12}$.

Dentre os profissionais que compõem a equipe que atua na ESF, o PEF pode ser considerado como o mais indicado para desenvolver, planejar e avaliar atividade física na atenção básica à saúde ${ }^{20}$. Entretanto, alguns aspectos ainda precisam ser esclarecidos, dentre os quais merecem destaque: será que as competências e habilidades 
do PEF são suficientes para atuar nesse âmbito? Esse profissional está preparado para trabalhar em equipes multiprofissionais? Como o PEF tem sido inserido e vem atuando nesse âmbito? Estas questões levam à reflexão sobre a formação desse profissional focando um perfil voltado para a atuação na atenção básica à saúde ${ }^{20-23}$. Diante desse contexto, esta pesquisa teve como objetivo analisar estudos originais sobre a inserção e atuação do PEF na atenção básica à saúde no Brasil.

\section{MÉTOdO}

Trata-se de um estudo de revisão sistemática que considerou os seguintes aspectos: i) processo de inserção do PEF na atenção básica à saúde; ii) atividades desenvolvidas pelos $\mathrm{PEF}$; iii) principais potencialidades e fragilidades na atuação do $\mathrm{PEF}$ na atenção básica. Esta revisão levou em consideração estudos originais sobre a inserção e atuação do PEF na atenção básica à saúde no Brasil, publicados entre janeiro de 2005 e outubro de 2011, em português ou inglês. A explicação para considerar apenas as publicações de 2005 em diante deve-se ao fato de que foi nesse ano que o Governo Federal do Brasil iniciou a liberação de recursos para os projetos de atividade física no SUS ${ }^{24}$. As buscas foram realizadas nas bases eletrônicas LILACS, MEDLINE, COCHRANE, no Google acadêmico e nas listas de referências dos artigos localizados, sendo utilizados os seguintes descritores: "saúde pública", "educação física”, "atenção básica à saúde”, "programa saúde da família”; e os termos "estratégia saúde da família", "núcleo de apoio à saúde da família" e "profissional de educação física". Os operadores lógicos and, or, not foram usados para combinar os descritores e termos utilizados na busca dos artigos.

A seleção dos estudos foi realizada conforme as seguintes etapas: i) leitura dos títulos dos artigos; ii) leitura dos resumos; iii) leitura na íntegra dos artigos selecionados a partir dos resumos. Todas as etapas foram efetuadas por dois revisores independentes e em caso de discordâncias um terceiro revisor foi consultado. A figura 1 apresenta as etapas do processo de revisão. Foram excluídos artigos de revisão, relatórios de pesquisa, teses, dissertações, capítulos ou livros, artigos de opinião de especialistas e trabalhos publicados em anais de eventos científicos.

Nos artigos selecionados para análise foram extraídos os seguintes itens: i) autor, ano e local do estudo; ii) periódico; iii) objetivos do estudo; iv) método e instrumento utilizado na coleta de dados; v) forma de inserção do PEF na atenção básica à saúde; vi) atuação do $\mathrm{PEF}$ em relação às atividades desenvolvidas; vii) principais potencialidades e fragilidades na atuação do PEF na atenção básica à saúde.

\section{RESULTADOS}

Considerando todas as fontes de busca, foram identificados 5.793 estudos. Com base na leitura dos títulos, 186 estudos foram selecionados e tiveram os resumos lidos. Nessa fase da revisão, mais quatro estudos identificados nas referências dos artigos, atenderam aos critérios de inclusão e foram lidos na íntegra. Dos 34 artigos que foram lidos na íntegra, nove foram selecionados para análise final (Figura 1).

A maioria dos estudos analisados foi publicada a partir de $2010^{10,20,23,25-27}$ (75\%, $\mathrm{n}=6$ ). Como pode ser observado no quadro 1 houve predominância de publicações na região Sudeste $(n=5)$, seguidas das regiões Sul $(n=2)$ e Centro-Oeste $(n=2)$. Nenhum estudo na região Norte e Nordeste do país foi localizado. A Revista Brasileira de Atividade Física e Saúde publicou o maior número de estudos sobre o tema $(75 \%, n=6)$. 
Busca nas bases eletrônicas

Janeiro de 2005 a outubro de 2011

Estudos identificados $(n=5.793)$

LILACS

633

MEDLINE

23

COCHRANE

1122

GOOGLE ACADÊMICO $\quad 4.015$

Estudos excluídos com base na

leitura dos títulos $(n=5.607)$

Estudos selecionados para leitura dos

resumos $(n=186)$

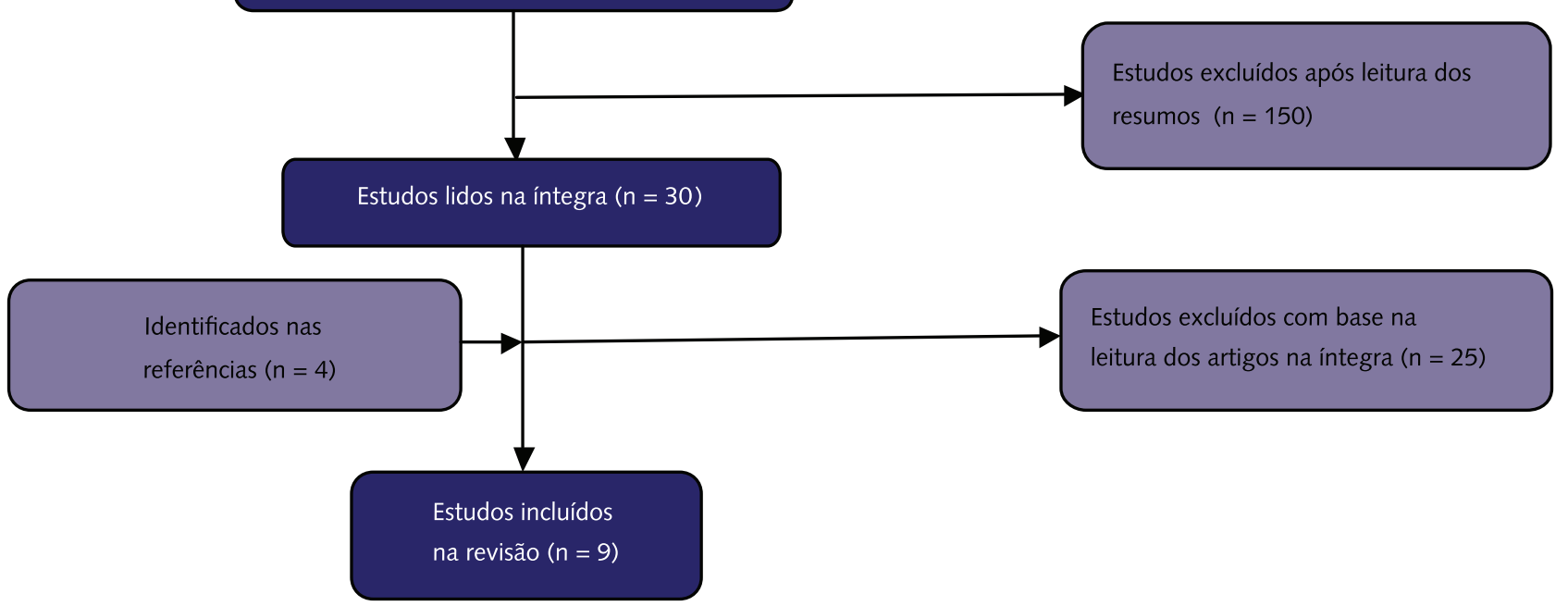

Figura 1 - Fluxograma das etapas do processo de revisão dos estudos.

Os objetivos dos estudos analisados consistiram em: apresentar as diretrizes gerais construídas pela experiência para intervenção na atenção básica à saúde; desenvolver, implementar e avaliar o impacto da atividade física em projetos de intervenção; descrever as ações do Ministério da Saúde, desde a aprovação da Política Nacional de Promoção da Saúde e a priorização da atividade física até ações de educação em saúde e capacitação; explicitar a introdução de novas contribuições do PEF ao controle da hanseníase através da difusão e incentivo ao aumento dos níveis de atividade física; descrever o funcionamento e o modelo lógico de um programa de intervenção com atividade física; analisar a atuação do PEF na ESF e verificar as características da intervenção do PEF no NASF (Quadro 2).

Observa-se que houve predominância de uso do método qualitativo $(n=7)$ nas pesquisas que envolviam programas de intervenção com atividade física ${ }^{20,23-28}$. Os demais estudos $(\mathrm{n}=2)$ utilizaram o método quantitativo ${ }^{10,16}$ para desenvolvimento das pesquisas. Pode-se observar que a entrevista e o questionário foram os instrumentos mais utilizados na coleta de dados ${ }^{23,27,16,28}$, além de pedômetro, escala de Borg e esfigmomanômetro ${ }^{10,20,28}$.

Quanto aos aspectos relacionados à inserção do PEF na atenção básica à saúde, observou-se que apenas 33,4\% ( $n=3)$ dos estudos analisados abordam esse aspecto $^{19,23,26}$. Foi verificado que a inserção do $\mathrm{PEF}$ ocorre predominantemente por meio 
de contrato temporário ${ }^{23,26}$ e em menor proporção, via concurso público $20,24,27$.

Quadro 1 - Resumo das informações sobre autor, periódico e metodologia de estudos a cerca da atuação do PEF na atenção básica à saúde, Brasil, 2011

\begin{tabular}{|c|c|c|c|}
\hline Autor (ano) & Local & Periódico & Método/Instrumento \\
\hline Kokubun et al. (2007) & Rio Claro / SP & $\begin{array}{l}\text { Revista Brasileira de Atividade } \\
\text { Física e Saúde }\end{array}$ & $\begin{array}{l}\text { Pesquisa qualitativa em programa de intervenção com } \\
\text { AF / escala de Borg, pedômetro, questionário SF- } 36 \text {. }\end{array}$ \\
\hline $\begin{array}{l}\text { Gomes; Duarte } \\
\text { (2008) }\end{array}$ & Florianópolis/SC & $\begin{array}{l}\text { Revista Brasileira de Atividade } \\
\text { Física e Saúde }\end{array}$ & $\begin{array}{l}\text { Estudo descritivo desenvolvimental com pré e pós- } \\
\text { teste em programa de intervenção com AF, com grupo } \\
\text { controle / Questionário. }\end{array}$ \\
\hline Malta et al. (2008) & Brasília & $\begin{array}{l}\text { Revista Brasileira de Atividade } \\
\text { Física e Saúde }\end{array}$ & Qualitativo, descritivo /*. \\
\hline Oliveira et al (2010) & Ipatinga/ MG & $\begin{array}{l}\text { Revista Brasileira de } \\
\text { Hipertensão }\end{array}$ & $\begin{array}{l}\text { Pesquisa quantitativa em programa de intervenção } \\
\text { com AF, com pré e pós-teste / Esfigmomanômetro } \\
\text { aneroide. }\end{array}$ \\
\hline $\begin{array}{l}\text { Nakamura; Chiyoda; } \\
\text { Gomes et al. (2010) }\end{array}$ & Rio Claro/SP & $\begin{array}{l}\text { Revista Brasileira de Atividade } \\
\text { Física e Saúde }\end{array}$ & Pesquisa descritiva com abordagem qualitativa / *. \\
\hline $\begin{array}{l}\text { Castro; Gonçalves } \\
\text { (2010) }\end{array}$ & Campinas/ SP & $\begin{array}{l}\text { Revista da Faculdade de } \\
\text { Educação Física da UNICAMP }\end{array}$ & $\begin{array}{l}\text { Pesquisa qualitativa em programa de intervenção com } \\
\text { AF / avaliação dermatológica. }\end{array}$ \\
\hline $\begin{array}{l}\text { Silva; Matsudo; Lopes } \\
\text { (2011) }\end{array}$ & São Caetano do Sul/SP & $\begin{array}{l}\text { Revista Brasileira de Atividade } \\
\text { Física e Saúde }\end{array}$ & $\begin{array}{l}\text { Pesquisa quali-quantitativa em programa de } \\
\text { intervenção com AF / Pedômetro. }\end{array}$ \\
\hline $\begin{array}{l}\text { Françasso, } \\
\text { Izidoro e Ribas (2011) }\end{array}$ & Campo Grande / MS & $\begin{array}{l}\text { SCRIBD http://pt.scribd.com/ } \\
\text { doc/55746451/ }\end{array}$ & Pesquisa qualitativa / Entrevista. \\
\hline $\begin{array}{l}\text { Souza e } \\
\text { Loch (2011) }\end{array}$ & $\begin{array}{l}\text { Apucarana, Cambé, } \\
\text { Ibiporã, Rolândia / PR }\end{array}$ & $\begin{array}{l}\text { Revista Brasileira de Atividade } \\
\text { Física e Saúde }\end{array}$ & $\begin{array}{l}\text { Pesquisa com abordagem qualitativa do tipo } \\
\text { exploratório-descritivo / Entrevista. }\end{array}$ \\
\hline
\end{tabular}

$\mathrm{PEF}=$ Profissional de Educação Física; AF = Atividade Física, UBS = Unidade Básica de Saúde; * Não informado.

Quadro 2 - Resumo dos estudos quanto aos objetivos, atividades, inserção, potencialidades e fragilidades do PEF na atenção básica à saúde, Brasil, 2011

\begin{tabular}{|c|c|c|c|c|}
\hline Autor / Ano & Objetivos relacionados & $\begin{array}{l}\text { Forma de } \\
\text { Inserção do PEF }\end{array}$ & Atuação do PEF - Atividades & $\begin{array}{l}\text { Potencialidades / } \\
\text { Fragilidades }\end{array}$ \\
\hline $\begin{array}{l}\text { Kokubun et al. } \\
\text { (2007) }\end{array}$ & $\begin{array}{l}\text { Compartilhar as evidências } \\
\text { acumuladas ao longo de seis anos } \\
\text { de existência de um programa de } \\
\text { atividade física desenvolvido em UBS } \\
\text { no município de Rio Claro-SP, bem } \\
\text { como apresentar as diretrizes gerais } \\
\text { que norteiam essa intervenção direta. }\end{array}$ & * & $\begin{array}{l}\text { Orientação de exercícios } \\
\text { predominantemente } \\
\text { cardiorrespiratórios } \\
\text { (caminhadas e atividades } \\
\text { lúdicas) e exercícios } \\
\text { neuromotores (força, } \\
\text { agilidade, equilíbrio, } \\
\text { flexibilidade e coordenação). }\end{array}$ & $\begin{array}{l}\text { Redução dos níveis de } \\
\text { pressão sanguínea em } \\
\text { hipertensos, podendo ser } \\
\text { motivação para participação } \\
\text { em programas de atividade } \\
\text { física / * }\end{array}$ \\
\hline $\begin{array}{l}\text { Gomes; Duarte } \\
\text { (2008) }\end{array}$ & $\begin{array}{l}\text { Desenvolver, implementar e avaliar } \\
\text { uma intervenção de aconselhamento } \\
\text { sobre AF para promoção da saúde em } \\
\text { adultos, atendidos pela ESF, na cidade } \\
\text { de Florianópolis (Brasil). }\end{array}$ & * & $\begin{array}{l}\text { Aconselhamento para prática } \\
\text { de AF por meio de palestras e } \\
\text { cartilhas. }\end{array}$ & $\begin{array}{l}\text { Componente lúdico como } \\
\text { facilitador para participação } \\
\text { e aderência às atividades } \\
\text { * }^{*} \text {. }\end{array}$ \\
\hline $\begin{array}{l}\text { Malta et al. } \\
(2008)\end{array}$ & $\begin{array}{l}\text { Descrever ações da aprovação da } \\
\text { Política Nacional de Promoção } \\
\text { da Saúde e a priorização da AF, } \\
\text { o financiamento de projetos de } \\
\text { AF em municípios, a avaliação e } \\
\text { monitoramento dos mesmos, a } \\
\text { organização da vigilância dos fatores } \\
\text { de risco e protetores das DCNT, ações } \\
\text { de educação em saúde e capacitação. }\end{array}$ & $\begin{array}{l}\text { Não especifica } \\
\text { diretamente. } \\
\text { Vislumbra } \\
\text { contrato } \\
\text { temporário. }\end{array}$ & $* / *$ & $* / *$ \\
\hline
\end{tabular}




\begin{tabular}{|c|c|c|c|c|}
\hline $\begin{array}{l}\text { Oliveira et al } \\
(2010)\end{array}$ & $\begin{array}{l}\text { Avaliar o efeito de um programa de } \\
\text { exercício físico e sua adesão: 1) no } \\
\text { controle da hipertensão pelo tratamento } \\
\text { medicamentoso, 2) nos níveis de PA e } \\
\text { 3) na qualidade de vida de pacientes } \\
\text { hipertensos atendidos no Programa de } \\
\text { Saúde da Família (PSF) e Núcleo de } \\
\text { Apoio à Saúde da Família (NASF). }\end{array}$ & * & $\begin{array}{l}\text { Avaliação física e de qualidade } \\
\text { de vida; caminhada. }\end{array}$ & $\begin{array}{l}\text { Redução dos níveis de } \\
\text { pressão sanguínea em } \\
\text { hipertensos, podendo ser } \\
\text { motivação para participação } \\
\text { em programas de AF } /{ }^{*} \text {. }\end{array}$ \\
\hline $\begin{array}{l}\text { Nakamura; } \\
\text { Chiyoda; } \\
\text { Gomes et al. } \\
(2010)\end{array}$ & $\begin{array}{l}\text { Descrever o histórico, funcionamento } \\
\text { e o modelo lógico do programa de } \\
\text { intervenção "Saúde Ativa Rio Claro" } \\
\text { realizada no município de Rio Claro } \\
\text { no Estado de São Paulo, Brasil }\end{array}$ & * & $\begin{array}{l}\text { As atividades são planejadas } \\
\text { de acordo com os } \\
\text { princípios de periodização } \\
\text { do treinamento físico } \\
\text { organizados em ciclos de } \\
4 \text { semanas que englobam } \\
\text { exercícios cardiorrespiratórios } \\
\text { e neuromotores. Avaliação } \\
\text { das capacidades funcionais } \\
\text { e da composição corporal, } \\
\text { palestras e atividades lúdicas. }\end{array}$ & $\begin{array}{l}\text { O PEF é importante para } \\
\text { divulgação dos benefícios } \\
\text { da } A F^{*} / \text { dificuldade no } \\
\text { controle de reavaliação pelo } \\
\text { não comparecimento dos } \\
\text { alunos. }\end{array}$ \\
\hline $\begin{array}{l}\text { Castro; } \\
\text { Gonçalves } \\
\text { (2010) }\end{array}$ & $\begin{array}{l}\text { Explicitar a introdução de novas } \\
\text { contribuições do PEF ao controle da } \\
\text { hanseníase a nível local, operando } \\
\text { a partir da integração entre } \\
\text { academia e serviços. }\end{array}$ & * & $\begin{array}{l}\text { Treino sensorial, dança, } \\
\text { movimentos rítmicos, } \\
\text { alongamento muscular e } \\
\text { percepção sinestésica. }\end{array}$ & $\begin{array}{l}\text { Evidências a favor da } \\
\text { participação do PEF no } \\
\text { interior das equipes de } \\
\text { Saúde incumbidas de } \\
\text { prevenção e controle } \\
\text { de afecções, face à } \\
\text { especificidade das } \\
\text { habilidades desenvolvidas } \\
\text { em sua formação específica } \\
{ }^{*} \text {. }\end{array}$ \\
\hline $\begin{array}{l}\text { Silva; Matsudo; } \\
\text { Lopes (2011) }\end{array}$ & $\begin{array}{l}\text { Difundir e incentivar o aumento dos } \\
\text { níveis de atividade física. }\end{array}$ & $\begin{array}{l}\text { Concurso } \\
\text { Público }\end{array}$ & $\begin{array}{l}\text { Força muscular; Equilíbrio; } \\
\text { Potência aeróbia; } \\
\text { Flexibilidade. }\end{array}$ & $\begin{array}{l}\text { Redução dos níveis de } \\
\text { pressão sanguínea em } \\
\text { hipertensos, podendo ser } \\
\text { motivação para participação } \\
\text { em programas de AF; } \\
\text { Facilitar a promoção da } \\
\text { saúde / sobrecarga de } \\
\text { trabalho. }\end{array}$ \\
\hline $\begin{array}{l}\text { Francasso, } \\
\text { Izidoro e Ribas } \\
\text { (2011) }\end{array}$ & $\begin{array}{l}\text { Analisar a atuação do PEF inserido na } \\
\text { ESF através do projeto "Viver Legal" } \\
\text { na cidade de Campo Grande - MS. }\end{array}$ & Por contrato & $\begin{array}{l}\text { Caminhada, Ginástica, } \\
\text { Lian-gong, alongamento, } \\
\text { fortalecimento muscular, } \\
\text { avaliação física, aferição da } \\
\text { pressão arterial, capoeira, } \\
\text { atividades lúdicas, palestras } \\
\text { educativas, métodos } \\
\text { participativos (dinâmicas) e } \\
\text { ginástica laboral. }\end{array}$ & $\begin{array}{l}\text { Reconhecimento } \\
\text { da importância do } \\
\text { trabalho do PEF / falta } \\
\text { de treinamento; não } \\
\text { participar do planejamento } \\
\text { junto a equipe por } \\
\text { incompatibilidade de } \\
\text { horário; remuneração da } \\
\text { professora inferior a do } \\
\text { professor; sobrecarga de } \\
\text { trabalho. }\end{array}$ \\
\hline $\begin{array}{l}\text { Souza e } \\
\text { Loch (2011) }\end{array}$ & $\begin{array}{l}\text { Verificar as características de } \\
\text { intervenção dos PEF inseridos no } \\
\text { NASF. }\end{array}$ & * & $\begin{array}{l}\text { Exercícios de alongamentos, } \\
\text { fortalecimento muscular, } \\
\text { caminhada, e em menor } \\
\text { proporção exercícios de } \\
\text { coordenação motora e } \\
\text { atividades lúdicas; mini- } \\
\text { palestras sobre a importância } \\
\text { da AF. }\end{array}$ & $\begin{array}{l}\text { Ser um facilitador, } \\
\text { aumentando as chances } \\
\text { de tornar as pessoas } \\
\text { fisicamente ativas / } \\
\text { necessidades de ajustes na } \\
\text { formação para o trabalho } \\
\text { em equipes de saúde. }\end{array}$ \\
\hline
\end{tabular}

$\mathrm{PEF}=$ Profissional de Educação Física; UBS= Unidade Básica de Saúde; AF = Atividade Física* Não informado; ESF= Estratégia Saúde da Família; AF= Atividade física; NASF= Núcleo de Apoio à Saúde da Família 
Sobre as atividades desenvolvidas pelo PEF na sua atuação na ESF, verificou-se que as intervenções com os usuários são coletivas, com abordagens multiprofissionais e interdisciplinares voltadas principalmente para pessoas com DCNT 5,10, 20, 23, 25, 28 . A caminhada ${ }^{10,23,27,28}$, alongamento ${ }^{23,26,27}$, fortalecimento muscular ${ }^{20,23,27,28}$, equilíbrio ${ }^{20,28}$, palestras ${ }^{16,25,27}$ e avaliação das capacidades funcionais e da qualidade de vida $^{10,25}$ e atividades lúdicas ${ }^{23,25,27,28}$ foram as atividades mais realizadas. No trabalho em equipe, a maior interação do PEF se dá com o agente comunitário de saúde (ACS) e com o enfermeiro, mas raramente com o médico ${ }^{20,28}$.

As potencialidades referentes à atuação do $\mathrm{PEF}$ identificadas nos estudos analisados foram: i) desenvolver as atividades físicas/práticas corporais nesse âmbito ${ }^{26}$, ${ }^{27}$; ii) facilitar para que as pessoas se tornem fisicamente ativas ${ }^{23,27}$; iii) contribuir por meio da sua atuação para melhoria dos níveis de saúde e qualidade de vida da população $0^{10,20,28,29}$. As fragilidades identificadas foram: i) déficit na formação profissional em relação à atuação na saúde pública e no trabalho multiprofissional em saúde ${ }^{23}$; ii) dificuldades para o trabalho em equipe ${ }^{23}$; iii) sobrecarga de trabalho; iv) dificuldade para reavaliar os usuários devido ao fato de alguns abandonarem o programa antes da reavaliação ou faltar a mesma ${ }^{25}$.

\section{DISCUSSÃO}

Este estudo revisou a literatura sobre a inserção e atuação do PEF na atenção básica à saúde no Brasil. Constatou-se que há uma grande escassez de estudos sobre a atuação do PEF na atenção básica à saúde nos diversos Estados brasileiros, sobretudo nas regiões Norte e Nordeste do Brasil. O porquê de os estudos sobre essa temática se concentrarem na região Sudeste pode ser explicado pelo fato de que os primeiros grupos de pesquisa, a estudarem esse tema, estarem concentrados nessa região e por agregarem a maior quantidade de programas de Pós-Graduação em educação física. Destaca-se que nenhum artigo foi encontrado na língua inglesa. Acredita-se que isso se deva ao fato de outros países não adotarem o mesmo modelo de saúde brasileiro e por ser um tema relativamente recente e possivelmente de maior interesse para pesquisadores nacionais.

Em relação aos objetivos dos estudos sobre o PEF, verificou-se que eles passaram da simples descrição de diretrizes construídas pela experiência nas atividades práticas e da avaliação de intervenção com atividade física para: i) a descrição da atividade física como prioridade das Políticas Públicas de Saúde; ii) a contribuição do PEF na promoção da saúde, na difusão e no incentivo ao aumento dos níveis de atividade física; iii) a verificação e análise da atuação do PEF na ESF/ NASF. Isso pode ser explicado pelo fato de que as atividades desenvolvidas pelo PEF na atenção básica à saúde passaram a ser regidas por diretrizes do Ministério da Saúde ${ }^{28}$, e pela verificação ${ }^{23}$ e avaliação da intervenção ${ }^{10,16}$. Essa sequência dos objetivos dos estudos analisados apresenta elementos que contribuem com a construção de informações sólidas que fortalecem a inclusão do PEF na atenção básica à saúde.

O método de pesquisa qualitativa tem sido uma novidade nos estudos da atividade física, pois, normalmente, são mais frequentemente utilizados nas áreas da antropologia, educação e sociologia ${ }^{30}$. Os instrumentos mais utilizados na coleta de dados dos estudos analisados foram a entrevista e o questionário, que são típicos das pesquisas qualitativas $23,2716,28$. Isso pode ser explicado pela versatilidade desses instrumentos na aquisição de respostas a questionamentos de uma pesquisa, seja ela qualitativa ou quantitativa. Também é importante considerar a natureza do 
problema em estudo sobre esse tema, requerendo mais informações qualitativas do que quantitativas.

A história da inserção não oficial (sem leis e portarias reguladoras) do PEF na ESF data do ano 2000 na cidade de Sobral no Ceará, fato proporcionado através da Secretaria de Desenvolvimento Social e da Saúde ${ }^{27}$. Posteriormente em 2005, a partir da seleção de projetos de atividade física no SUS, por meio de edital, o Governo Federal deu apoio financeiro com repasse para as 27 capitais brasileiras ${ }^{24}$. Isso favoreceu um aumento expressivo no número de $\mathrm{PEF}$ em ações de promoção e cuidados com a saúde e prevenção de doenças. Apesar de ter sido oficialmente proposta em 2008, a inserção do PEF, conforme a portaria G.M nº. 154/2008 do Ministério da Saúde ${ }^{14}$, só recentemente vem sendo implementada em vários municípios, a exemplo de Rio Claro-SP, Florianópolis-SC e Campo Grande-MS. Tomando como base o princípio de promoção da saúde, os profissionais de saúde de categorias distintas da educação física se mostram favoráveis à inserção do $\mathrm{PEF}$ na atenção básica à saúde ${ }^{8}$. Verificou-se neste estudo que a inserção do PEF na atenção básica à saúde ocorre por intermédio das secretárias dos municípios, sobretudo na forma de contrato temporário ${ }^{24,27}$, não oferecendo estabilidade profissional e um possível comprometimento da continuidade de suas ações. Por outro lado, se a inserção ocorre por meio de concurso público, os profissionais contratados terão estabilidade e, por conseguinte, será mais provável a continuidade do trabalho planejado. Isso também permitirá avaliar de forma mais consistente, tanto o progresso dos usuários quanto as suas próprias ações na atenção básica à saúde.

Os resultados desta revisão indicaram que a atuação do PEF ocorre predominantemente com pessoas portadoras de DCNT ou idosas ${ }^{20,28}$. A caminhada ${ }^{8,19,23,}$ ${ }^{24}$, o fortalecimento muscular ${ }^{20,23,27,28}$ e as atividades lúdicas ${ }^{23,25,27,28}$ foram as práticas de atividade física mais realizadas. Essas atividades se destacam possivelmente por serem de baixo custo, de maior facilidade quanto aos locais de prática, pois não exigem espaços específicos e por seus efeitos na melhoria das condições de saúde dos seus praticantes, são de baixa complexidade podendo ser realizadas por pessoas com baixos níveis de aptidão física. Os exercícios para fortalecimento muscular têm sido bastante empregados em grupos de idosos para evitar quedas, e o alongamento objetivando tanto a preparação para atividade quanto para reduzir as perdas de flexibilidade produzidas pelo envelhecimento ${ }^{28}$. Outras ações relatadas pelos estudos foram o uso de palestras e cartilhas pelo PEF no aconselhamento para prática de atividade física ${ }^{16}$. Todas essas intervenções são especialmente importantes, pois alcançam um grande número de pessoas, considerando que a divulgação de informações sobre os benefícios da atividade física pode contribuir para a adoção de um estilo de vida ativo e saudável ${ }^{24,28}$.

A intervenção do PEF através de um programa de atividade física em Rio Claro-SP, realizada com grupos de hipertensos, diabéticos e obesos, entre os anos de 2001 e 2007, demonstrou a importância do PEF na atuação interdisciplinar e multiprofissional que acontecia com enfermeiras e médicos ${ }^{25,28}$. Além disso, outro estudo $^{20}$ relata que os ACS são os que mais desenvolvem trabalho interdisciplinar com o PEF.

Ao analisar alguns estudos desta revisãa ${ }^{16,20,25}$, percebeu-se que a atuação do PEF na atenção básica à saúde tem buscado cumprir as diretrizes do NASF e a portaria no 2.488/2011 do Ministério da Saúde, ou seja, o PEF deve desenvolver suas ações com intuito de promoção, reabilitação e manutenção da saúde, priorizando os grupos de risco com o objetivo de prevenir o aparecimento ou a persistência de doenças e danos evitáveis ${ }^{15,31}$. 
Os resultados deste estudo indicam que o PEF na atenção básica à saúde tem suas potencialidades no sentido de ser considerado o mais capaz para orientar atividades físicas ${ }^{26,27}$. Essa potencialidade pode ser verdadeira, apesar das deficiências na sua formação. O PEF é um facilitador para aumentar as chances dos indivíduos serem fisicamente ativos ${ }^{23,27}$. Isso gera benefícios adquiridos com a prática de atividade física orientada ${ }^{10,20,28,29}$, bem como, conduz os demais profissionais da ESF e gestores a reconhecerem o importante papel desse profissional na promoção e reabilitação da saúde e na prevenção de doenças.

O PEF tem fragilidades em sua formação que não é direcionada ao trabalho multiprofissional em equipes de saúde ${ }^{23,27}$, ocorrendo a falta de experiências teórico - praticas anteriores (treinamento prévio) a sua atuaçãa ${ }^{27}$. Isso dificulta a quebra do paradigma do atendimento individual para as propostas das ações coletivas e nas realizações de ações multidisciplinares e interdisciplinares ${ }^{23}$. Nesse sentido, por possuir maior número de disciplinas relacionadas ao tema, o curso de Bacharelado em Educação Física oferece melhor formação para atuação na saúde do que o de licenciatura $^{22}$. Para suprir o déficit da graduação é necessário que novos cursos de PósGraduação nesta área sejam ofertados para melhor qualificação do $\mathrm{PEF}^{32}$. Quanto à sobrecarga de trabalho do PEF, na maioria das vezes, ela ocorre devido a atuação semanal em várias UBS, e por ser o único da equipe que chega a atender 11 bairros $^{20}$ e atuar em até $5 \mathrm{UBS}^{23}$. Outra fragilidade é a dificuldade em reavaliar os usuários do serviço devido às faltas e/ou desistências dos mesmos, bem como em controlar a intensidade das atividades desenvolvidas nesse âmbito ${ }^{25}$. Contudo, a escala de Borg é sugerida como um instrumento eficaz no controle da intensidade da atividade física $^{28}$. Esses fatores representam os maiores desafios a serem enfrentados.

Conclui-se que a inserção do PEF tem ocorrido predominantemente por meio de contrato temporário. As atividades mais desenvolvidas na atuação do PEF são caminhada, fortalecimento muscular e atividades lúdicas. As potencialidades desse profissional consistem em ser visto pelos outros profissionais das equipes como o mais capaz para desenvolver as atividades físicas/práticas corporais na atenção básica à saúde, além de ser um facilitador para as pessoas se tornarem fisicamente ativas e contribuir para melhoria da qualidade de vida. Quanto às fragilidades, uma das preocupações foi a de que a atual formação do PEF na licenciatura não contempla adequadamente os conhecimentos necessários nesse campo de atuação.

Percebe-se que algumas mudanças têm ocorrido na formação inicial do Bacharelado em Educação Física, com currículos mais direcionados às disciplinas que contemplem uma formação no sentido de fomentar as competências e habilidades necessárias para atuação na atenção básica à saúde. Apesar das dificuldades, o PEF é o único da equipe do NASF com formação voltada para orientar a prática de atividades físicas. Esse profissional tem função relevante no cumprimento dos princípios do SUS em relação à política de saúde e de promoção da atividade física no Brasil. Sugere-se que mais estudos sobre as intervenções dos PEF na atenção básica à saúde sejam desenvolvidos para servir como referências na atuação profissional, bem como para análise e avaliação dos serviços nesse âmbito.

\section{REFERÊNCIAS}

1. Junqueirai TDS, Cotta RMM, Gomes RC, Silveira SDFR, Siqueira-Batista R, Pinheiro TMM, Melo EMD. Saúde, democracia e organização do trabalho no contexto do Programa de Saúde da Família: desafios estratégicos. Rev Bras Educ Med 2009; 33: 122-133. 
2. Brasil, Ministério da Saúde. Lei 8.880 de 19 de setembro de 1990. Dispõe sobre as condições para a promoção, proteção e recuperação da saúde, a organização e o funcionamento dos serviços correspondentes e dá outras providências. Brasilia/DF: Ministério da Saúde, 1990.

3. Ferreira MEV, Schimith MD, Cáceres NC. Necessidades de capacitação e aperfeiçoamento dos profissionais de equipes de saúde da família da 4a Coordenadoria Regional de Saúde do Estado do Rio Grande do Sul. Ciên Saúde Col 2010; 15: 2611-2620.

4. Leite RFB, Veloso TMG. Limites e avanços do Programa Saúde da Família de Campina Grande: um estudo a partir de representações sociais. Saúde e Soc 2009; 18: 50-62.

5. Rocha PDM, Uchoa ADC, Rocha NDSPD, Souza ECFD, Rocha MDL, Pinheiro TXDA. Avaliação do Programa Saúde da Família em municípios do Nordeste brasileiro: velhos e novos desafios. Cad Saúde Pública 2008; 24: 69-78.

6. Ronzani TM, Silva CDM. O Programa Saúde da Família segundo profissionais de saúde, gestores e usuários. Ciên Saúde Col 2008; 13: 23-34.

7. Brasil. Ministério Da Saúde. Portaria G.M. n. 692, de 25/03/94; cria o Programa de Interiorização do SUS e o programa de Saúde da Família, com códigos específicos na tabela do SIA/SUS. Diário Oficial da União. Brasília, Governo Federal: Ministério da Saúde, 1994.

8. Miranda FM, Melo RVD, Raydan FPS. A inserção do profissional de Educação Física no Programa de Saúde da Família segundo a opinião dos profissionais integrantes do programa em uma Unidade Básica de Saúde da cidade de Coronel Fabriciano - MG. Movimentum - Rev digit Edu Fís 2007; 2.

9. Conill EM. Ensaio histórico-conceitual sobre a Atenção Primária à Saúde: desafios para a organização de serviços básicos e da Estratégia Saúde da Família em centros urbanos no Brasil. Cad Saúde Pública 2008; 24: 7-27.

10. Oliveira KPC, Vieira EDL, Oliveira JDD, Oliveira KRD, Lopes FJG, Azevedo LF. Exercício Aeróbio no Tratamento da Hipertensão Arterial e Qualidade de Vida de Pacientes Hipertensos do Programa do Programa de Saúde de Ipatinga. Rev Bras Hipertens 2010; 17: 78-86.

11. Kantorski LP, Jardim VMDR, Pereira DB. A Integralidade no Cotidiano de Trabalho na Estratégia Saúde da Família. Rev Gaucha Enferm 2009; 30: 594-601.

12. Pinto NRS, Tanaka OY, Spedo SM. Política de saúde e gestão no processo de (re)construção do SUS em município de grande porte: um estudo de caso de São Paulo, Brasil. Cad Saúde Pública 2009; 25: 927-938.

13. Costa CM, Polignamo MV.Integralidade da Saúde no Programa de Saúde da Família: proposta de um indicador. Revista Médica de Minas Gerais 2008;18:19-24.

14. Brasil, Ministério Da Saúde. Portaria Ministerial no 154, de 24 de janeiro de 2008, Diário Oficial da União no 43, de 04/03/2008, Seção 1, fls. 38 a 42.

15. Brasil, Ministério Da Saúde. Portaria Ministerial no 2.488, de 21 de outubro de 2011. Diário Oficial da União no 204, 24 de outubro de 2011, seção 1, 2011: 48-65.

16. Gomes MDA, Duarte MDFDS. Efetividade de uma Intervenção de Atividade Física em Adultos Atendidos pela Estratégia de Saúde da Família: Programa Ação e Saúde Floripa Brasil. Rev Bras Ativ Fis Saúde 2008; 13: 44-56.

17. http://www.datasus.gov.br/conselho/reso197/res21897.htm/.Acessado em 08 de outubro de 2011.

18. Organização Mundial Da Saúde. Riscos Globais de Saúde: Mortalidade e Carga de Doenças Atribuíveis aos Riscos mais Graves. Genebra, Suiça: Organização Mundial de Saúde, 2009.

19. Pereira JC, Barreto SM, Passos VMDA. Perfil de risco cardiovascular e autoavaliação da saúde no Brasil: estudo de base populacional. Rev Panam Salud Publica 2009;25:491-498.

20. Silva L, Matsudo S, Lopes G. Do diagnóstico à ação: Programa comunitário de atividade física na atenção básica: a experiência do município de São Caetano do Sul, Brasil. Rev Bras Ativ Fis Saúde 2011; 16: 84-88.

21. Pasquim HM. A saúde coletiva nos cursos de graduação em Educação Física. Saúde e Soc 2010; 19: 193-200.

22. Anjos TCD, Duarte ACGDO. A Educação Física e a Estratégia de Saúde da Família: formação e atuação profissional. Physis: Rev Saúde Col 2009; 19: 1127-1144.

23. Souza SCD, Loch MR. Intervenção do profissional de educação física nos Núcleos de Apoio à Saúde da Família em municípios do norte do Paraná. Rev Bras Ativ Fis Saúde 2011; 16: 5-10.

24. Malta DC, Castro AMD, Cruz DKA, Gosh CS. A Promoção da Saúde e da Atividade Física no Sistema Único de Saúde. Rev Bras Ativ Fis Saúde 2008; 13: 24-27. 
25. Nakamura PM, Papini CB, Chiyoda A, Gomes GADO, Valdanha Netto A, TEIXEIRA IP, Luciano E, Kokubun E. Programa de intervenção para a prática de atividade física: Saúde Ativa Rio Claro. Rev Bras Ativ Fis Saúde 2010; 15: 128-132.

26. Castro GCD, Gonçalves A. Contribuições do Profissional da Educação Física no Controle da Hanseníase a Nível Local. Rev Fac Ed Fís da UNICAMP 2010; 8.

27. http://pt.scribd.com/doc/55746451/o-profissional-de-educaçao-fisica-e-a-estrategia-desaude-da-familia-na-cidade-de-campo-grande-ms/. Acessado em 06 de outubro de 2011.

28. Kokubun E, Luciana E, Sibuya CY, Queiroga MR, Ribeiro PAB, Silveira RF, Nakamura PM. Programa de Atividade Física em Unidades Básicas de Saúde: Relato de experiência no Município de Rio Claro-SP. Rev Bras Ativ Fis Saúde 2007; 12: 45-53.

29. Börjesson M, Dahlöf B. Physical activity has a key role in hypertension therapy. Lakartidningen 2005; 102: 123-4, 126, 128-9.

30. Thomas JR, Nelson SJ, Silverman. Métodos de Pesquisa em Atividade Física. ARTMED, 2007.

31. Brasil, Ministério da Saúde. Diretrizes do NASF - Núcleo de Apoio à Saúde da Família. Secretaria de Atenção a Saúde, Cadernos de Atenção Básica. Brasil, Brasilia, DF, 2010.

32. Florindo AA. Núcleos de Apoio à Saúde da Família e a Promoção das Atividades Físicas no Brasil: de onde viemos, onde estamos e para onde vamos. Rev Bras Ativ Fis Saúde 2009; 14: 72-73.

$$
\begin{array}{r}
\text { Endereço para Correspondência } \\
\text { José Damião Rodrigues } \\
\text { Universidade Federal da Paraíba - } \\
\text { UFPB, Centro de Ciências da Saúde } \\
\text { - CCS, Departamento de Educação } \\
\text { Física - DEF. Cidade Universitária, CEP } \\
\text { 58.059.900, João Pessoa, Paraíba, } \\
\text { Brasil. Telefone: (083) 3216-7030 } \\
\text { E-mail: prof.damiao@gmail.com }
\end{array}
$$

Recebido 29/05/2012

Revisado 28/07/2012

Aprovado 01/08/2012 